\title{
Unified formalism for excess current noise in random-walk models
}

\author{
Jeppe C. Dyre \\ Institute of Mathematics and Physics (IMFUFA), Roskilde University, Postbox 260, DK-4000 Roskilde, Denmark
}

(Received 23 September 1987; revised manuscript received 27 January 1988)

\begin{abstract}
Excess current noise in random-walk models with a frequency-independent conductivity is studied from a general point of view. By introducing a "dynamical" diffusion constant, it is shown that the current autocorrelation function in an external field probes the equilibrium dynamical diffusion-constant autocorrelation function. From this a number of results, previously shown for particular models, are derived. Also, it is shown that the external-field current autocorrelation function is proportional to the equilibrium autocorrelation function for the absolute value of the current. Thus, the excess-noise spectrum probes the equilibrium-speed autocorrelation function. In the treatment advanced here, the study of excess current noise in random-walk models reduces to a study of the stochastic point process constituted by the particle-jump times. This point process contains all information about the noise. As an illustration of the general theory, the continuous-time random-walk model is briefly reviewed and a simple derivation of the excess noise in the model is given. Finally, the role of Fermi statistics in models for $1 / f$ noise is discussed. It is argued that number-fluctuation models, i.e., models with long trapping times, are incompatible with Fermi statistics. On the other hand, it is shown there is a peculiar "single-particle" $1 / f$ noise which is due to Fermi statistics but has nothing to do with the observed $1 / f$ current noise.
\end{abstract}

\section{INTRODUCTION}

Electrical $1 / f$ noise has been a major puzzle in solidstate physics for many years and is still far from being understood. $^{1-11}$ This noise is found at low frequencies in apparently any conducting solid in an external electric field. $1 / f$ noise is always observed together with white noise, the origin of which is well understood, and therefore $1 / f$ noise is often referred to as excess noise. One may speak of excess current noise in a constant-voltage circuit or excess voltage noise in a constant-current circuit. The spectra of the two are always identical and only excess current noise will be discussed here.

Experimentally, the spectrum of excess current noise is given by

$$
S_{\mathrm{exc}, J}(\omega)=K \frac{\langle J\rangle_{E}^{2}}{V} \omega^{-\alpha},
$$

where $\langle J\rangle_{E}$ is the average current in the electric field $E$, $V$ is the volume of the sample, $K$ is a constant, and the exponent $\alpha$ is close to 1 . The case $\alpha=1$ has given the name to the subject: $1 / f$ noise, where $f$ is the frequency. The fact that the noise is proportional to $\langle J\rangle_{E}^{2}$ suggests it is the resistance that fluctuates and consequently one often speaks about $1 / f$ resistance fluctuations. If the resistance really fluctuates, however, there should be $1 / f$ fluctuations in the magnitude of the Nyquist noise in zero external field. This was shown actually to be the case by Voss and Clarke in $1976 .{ }^{12}$ Their work was a major breakthrough because it showed that $1 / f$ noise is an equilibrium phenomenon and is not created by the rather strong electric fields usually applied when measuring $1 / f$ excess noise. The Voss and Clarke experiment raised the obvious question: How can noise fluctuate, being itself due to fluctuations? It was soon shown that fluctuations in the magnitude of the Nyquist noise are due to nontrivial fourth-order correlations in the equilibrium current or voltage fluctuations, ${ }^{13,14}$ implying these fluctuations are non-Gaussian.

During the 1980's there has been considerable interest in random-walk models for $1 / f$ noise. These models are probably the simplest one can think of as a means of getting a better understanding of the purely statistical properties of the noise. In particular, the appearance of nonGaussian equilibrium current fluctuations can be studied in detail. In Sec. II of the present paper we study general features of the current noise in random-walk models. The treatment is centered around the concept of a "dynamical" diffusion constant. In Sec. III the continuous-time random-walk (CTRW) model for $1 / f$ noise is briefly reviewed as an illustration of the general theory. Finally, Sec. IV contains a discussion where the role of Fermi statistics for the application of randomwalk models is emphasized. It is argued that any model for $1 / f$ noise based on long trapping times, including the CTRW model in its multiple trapping realization, is incompatible with Fermi statistics and is therefore unrealistic.

\section{EXCESS CURRENT NOISE IN RANDOM-WALK MODELS}

To simplify the discussion we consider just one particle which performs a random walk in one dimension on a lattice with lattice constant $a$. No assumption is made about the underlying dynamics which does not have to be Markovian. It is assumed that the direction of any single jump is random; via the fluctuation-dissipation theorem this ensures a frequency-independent conductivity. If the 
particle jumps at times $\tau_{i}$ we assign to it a dynamical diffusion constant $D(t)$ defined by

$$
D(t)=\frac{a^{2}}{2} \sum_{i} \delta\left(t-\tau_{i}\right)
$$

$D(t)$ is characterized by

$$
\left\langle v(t) v\left(t^{\prime}\right)\right\rangle_{\mathrm{re}}=2 D(t) \delta\left(t-t^{\prime}\right),
$$

where $v$ is the velocity, or

$$
D(t)=\int_{0}^{\infty}\langle v(t) v(t+\tau)\rangle_{\mathrm{re}} d \tau
$$

Here, \langle\rangle$_{\text {re }}$ denotes a "restricted ensemble average" by which is meant an average over all trajectories with the same $D(t)$, i.e., with the same jump times. The time average of $D(t)$ is the ordinary diffusion constant $D$ :

$$
D=\langle D(t)\rangle=\lim _{T_{0} \rightarrow \infty} \frac{1}{T_{0}} \int_{0}^{T_{0}} D(t) d t .
$$

The relevance of $D(t)$ to the excess current noise becomes clear when the current in an external electric field is evaluated. In a weak field it is slightly more probable for the particle to jump to one side than to the other. To lowest order in $E$ the total jump probability does not change, however, and the particle still jumps at times $\tau_{i}$. The restricted ensemble average current is thus proportional to the equilibrium $D(t)$ :

$$
\langle J(t)\rangle_{E, \mathrm{re}}=\frac{q^{2} E}{k T} D(t),
$$

where $q$ is the particle charge, and $k$ and $T$ have their usual meaning. The constant of proportionality follows from requiring the time average of Eq. (6) to obey the Nernst-Einstein relation. The measured current noise is the cosine transform of the current autocorrelation function. Since the currents at different times within the restricted ensemble are uncorrelated, one has

$$
\begin{aligned}
\langle J(t) J(0)\rangle_{E, \mathrm{re}} & =\langle J(t)\rangle_{E, \mathrm{re}}\langle J(0)\rangle_{E, \mathrm{re}} \\
& =\left(\frac{q^{2} E}{k T}\right)^{2} D(t) D(0) \quad(t>0) .
\end{aligned}
$$

When averaged over the whole ensemble of possible jump times this leads to

$$
\langle J(t) J(0)\rangle_{E}=\left(\frac{q^{2} E}{k T}\right)^{2}\langle D(t) D(0)\rangle \quad(t>0),
$$

where \langle\rangle on the right-hand side denotes an equilibrium average. To obtain the total current autocorrelation function one should add to this expression a white-noise term proportional to $\delta(t)$. This term is not of interest here. According to Eq. (8) the excess noise measures the spectrum of dynamical diffusion constant fluctuations in equilibrium.

The autocorrelation function $\langle D(t) D(0)\rangle$ has a simple physical interpretation. From Eq. (2) it follows immediately that

$$
\langle D(t) D(0)\rangle=\frac{D a^{2}}{2} p(t \mid 0) \quad(t>0),
$$

where $p(t \mid 0)$ denotes the probability density for a jump at time $t$ given the particle jumped at $t=0$. Except for a numerical constant, low-frequency excess noise is thus the cosine transform of $p(t \mid 0) .^{15} 1 / f$ noise implies long-time correlations in this probability. The particle or the medium in which it jumps somehow has a long-term memory. For an ordinary random walk, on the other hand, $p(t \mid 0)$ is constant and there is no excess noise.

Generalizing the above results to more than one particle is straightforward. Assuming the particles are independent and noninteracting, one just lets $\tau_{i}$ denote the collection of jump times for all the particles. In generalizing to $d$ dimensions the factor 2 in Eq. (2), etc., should be replaced by $2 d$. Equations like (6) and (8) apply unchanged where $J$ is now, of course, the component of the current in the direction of the field. No new features appear and in the rest of the paper only the one-dimensional case will be considered. The important thing, which is independent of the dimension and number of particles, is that all information about the noise lies in the statistical properties of the collection of jump times $\tau_{i}$. In statistics a stochastic collection of times is referred to as a "point process." The study of point processes is a mature branch of the theory of stochastic processes. ${ }^{16-18}$ Point processes have been applied in the study of photoelectron statistics, cosmic-ray showers, kinetic theory, population growth, telephone traffic, etc. An important class of point processes is the class of so-called doubly stochastic Poisson processes. An example is the jump times $\tau_{i}$ for a particle performing a random walk where the jump probability at time $t, \gamma(t)$, is itself a stochastic process. Here one may define a second kind of time-dependent diffusion constant $\widetilde{D}(t)$ by

$$
\widetilde{D}(t)=\frac{a^{2}}{2} \gamma(t)
$$

While $\widetilde{D}(t)$ is of course different from $D(t)$, their statistical properties are identical: For the average over a particular realization of $\gamma(t)$ it is easy to see that $\left\langle D\left(t_{1}\right) \cdots D\left(t_{n}\right)\right\rangle_{\gamma(t)}=\widetilde{D}\left(t_{1}\right) \cdots \widetilde{D}\left(t_{n}\right)$. Averaging this expression over all possible $\gamma(t)$ 's leads to

$$
\left\langle D\left(t_{1}\right) \cdots D\left(t_{n}\right)\right\rangle=\left\langle\widetilde{D}\left(t_{1}\right) \cdots \widetilde{D}\left(t_{n}\right)\right\rangle .
$$

Thus, our definition of $D(t)$ in Eq. (2) is consistent in the case where the ordinary diffusion constant really does fluctuate in time via a time-dependent jump probability.

We now turn to the problem of expressing the current autocorrelation function in an external field in terms of equilibrium current fluctuations. Focusing attention on diffusion-constant fluctuations according to Eq. (8), we note that the integrated dynamical diffusion constant counts the number of jumps $N$ in time $t$ :

$$
N=\frac{2}{a^{2}} \int_{0}^{t} D\left(t^{\prime}\right) d t^{\prime}
$$

For the variance of $N$ one has 


$$
\begin{aligned}
\left\langle(\Delta N)^{2}\right\rangle_{t}= & \frac{4}{a^{4}}\left[\int_{0}^{t} \int_{0}^{t}\left\langle D\left(t^{\prime}\right) D\left(t^{\prime \prime}\right)\right\rangle d t^{\prime} d t^{\prime \prime}\right. \\
& \left.-\left[\int_{0}^{t}\left\langle D\left(t^{\prime}\right)\right\rangle d t^{\prime}\right]^{2}\right] \\
= & \frac{8}{a^{4}} \int_{0}^{t}(t-\tau) C_{D}(\tau) d \tau,
\end{aligned}
$$

where $C_{D}(\tau)$ is the autocorrelation function for the dynamical diffusion constant,

$$
C_{D}(\tau) \equiv\langle D(\tau) D(0)\rangle-D^{2}
$$

Equation (13) leads to

$$
C_{D}(t)=\frac{a^{4}}{8} \frac{d^{2}}{d t^{2}}\left\langle(\Delta N)^{2}\right\rangle_{t}
$$

The next step is to relate the right-hand side to averages of the displacement in time $t, \Delta x(t)$. The quantity $\left\langle e^{i k \Delta x(t)}\right\rangle$ is an average of a product of independent factors $e^{ \pm i k a}$ and thus

$$
\left\langle e^{i k \Delta x(t)}\right\rangle=\left\langle\cos ^{N}(k a)\right\rangle_{t} .
$$

From this equation averages of all powers of $\Delta x(t)$ can be found. For the first two nonzero averages one finds

$$
\begin{aligned}
\left\langle(\Delta x)^{2}(t)\right\rangle & =-\left.\frac{d^{2}}{d k^{2}}\left\langle\cos ^{N}(k a)\right\rangle_{t}\right|_{k=0}=a^{2}\langle N\rangle_{t} \\
\left\langle(\Delta x)^{4}(t)\right\rangle & =\left.\frac{d^{4}}{d k^{4}}\left\langle\cos ^{N}(k a)\right\rangle_{t}\right|_{k=0} \\
& =a^{4}\left(3\left\langle N^{2}\right\rangle_{t}-2\langle N\rangle_{t}\right) .
\end{aligned}
$$

In particular, the fourth-order cumulant of $\Delta x(t)$, $\left\langle\Delta x^{(4)}(t)\right\rangle \equiv\left\langle(\Delta x)^{4}(t)\right\rangle-3\left\langle(\Delta x)^{2}(t)\right\rangle^{2}$, is given by

$$
\left\langle\Delta x^{(4)}(t)\right\rangle=a^{4}\left[3\left\langle(\Delta N)^{2}\right\rangle_{t}-2\langle N\rangle_{t}\right] .
$$

Since $\langle N\rangle_{t}$ is always proportional to $t$, Eq. (18) in conjunction with Eq. (15) implies ${ }^{19-22}$

$$
C_{D}(t)=\frac{1}{24} \frac{d^{2}}{d t^{2}}\left\langle\Delta x^{(4)}(t)\right\rangle \text {. }
$$

Equation (19) was first derived by Kuzovlev and Bochkov as a consequence of

$$
\left\langle e^{i k \Delta x(t)}\right\rangle=\left\langle\exp \left(-k^{2} \int_{0}^{t} D\left(t^{\prime}\right) d t^{\prime}\right)\right\rangle,
$$

derived for "slowly fluctuating" $D(t){ }^{20}$ Their definition of $D(t)$ is not obvious, but Eq. (20) is not valid for any reasonable definition of $D(t)$ as long as $a>0$, since it does not apply even in the case of an ordinary random walk with a constant $D(t)$ : For $D(t)=D$ Eq. (20) reduces to $\ln \left\langle e^{i k \Delta x(t)}\right\rangle=-k^{2} D t$ which implies $\left\langle\Delta x^{(4)}(t)\right\rangle=0$. In reality $\left\langle\Delta x^{(4)}(t)\right\rangle \propto t$ in this case since cumulants are additive and $\Delta x(t)$ is a sum of independent increments. Instead, Eq. (20) must be replaced by

$\left\langle e^{i k \Delta x(t)}\right\rangle=\left\langle\exp \left[\frac{2 \ln [\cos (k a)]}{a^{2}} \int_{0}^{t} D\left(t^{\prime}\right) d t^{\prime}\right]\right\rangle$

which just combines Eqs. (12) and (16). For $a \rightarrow 0$ Eq. (21) reduces to Eq. (20). This limit, however, is only permissible in certain models. From Eq. (21) it is possible to derive Eq. (19) directly by expanding the logarithm of the equation to fourth order in $k$, but we found it more instructive and also useful below to arrive at Eq. (19) via averages of $N$ and its fluctuations.

Defining as usual the fourth-order cumulant of the velocity by

$$
\begin{aligned}
\left\langle v\left(t_{1}\right), v\left(t_{2}\right), v\left(t_{3}\right), v\left(t_{4}\right)\right\rangle \equiv & \left\langle v\left(t_{1}\right) v\left(t_{2}\right) v\left(t_{3}\right) v\left(t_{4}\right)\right\rangle-\left\langle v\left(t_{1}\right) v\left(t_{2}\right)\right\rangle\left\langle v\left(t_{3}\right) v\left(t_{4}\right)\right\rangle \\
& -\left\langle v\left(t_{1}\right) v\left(t_{3}\right)\right\rangle\left\langle v\left(t_{2}\right) v\left(t_{4}\right)\right\rangle-\left\langle v\left(t_{1}\right) v\left(t_{4}\right)\right\rangle\left\langle v\left(t_{2}\right) v\left(t_{3}\right)\right\rangle,
\end{aligned}
$$

Eq. (19) can be rewritten as

$$
C_{D}(t)=\int_{0}^{t} d \tau^{\prime} \int_{0}^{\tau^{\prime}} d \tau^{\prime \prime}\left\langle v(t), v\left(\tau^{\prime}\right), v\left(\tau^{\prime \prime}\right), v(0)\right\rangle
$$

The right-hand side is the Burnett coefficient. ${ }^{21,22}$ Equation (23) was derived by Kuzovlev and Bochkov ${ }^{20}$ from Eq. (20), and also by Nieuwenhuizen and Ernst from a different point of view. ${ }^{22}$ The latter authors define a "fluctuating diffusion coefficient," $D(t, \tau)$, by

$$
D(t, \tau)=\frac{1}{2} \frac{\partial}{\partial t}\left\{[x(t)-x(\tau)]^{2}\right\}
$$

They then proceed to show that for the Markovian random-walk model under study $C_{D} \equiv\left\langle D(t, \tau) D\left(t^{\prime}, \tau^{\prime}\right)\right\rangle-D^{2}$ is actually a function of $t-t^{\prime}$ only which obeys Eq. (23).

Combining Eqs. (8), (14), and (23) we finally arrive at

$$
\langle J(t) J(0)\rangle_{E}=\langle J\rangle_{E}^{2}+\left(\frac{E}{k T}\right)^{2} \int_{0}^{t} d \tau^{\prime} \int_{0}^{\tau^{\prime}} d \tau^{\prime \prime}\left\langle J(t), J\left(\tau^{\prime}\right), J\left(\tau^{\prime \prime}\right), J(0)\right\rangle \quad(t>0)
$$


Since cumulants are additive, this equation implies the excess current noise is proportional to $\langle J\rangle_{E}^{2} / V$ as in experiment [Eq. (1)]. Actually, this follows also directly from Eq. (8). It was first shown for a random-walk model by Tunaley. ${ }^{23}$ Equations like Eq. (25) have been derived by a number of authors, ${ }^{13,14,20-22,24}$ thereby greatly clarifying and simplifying the subject by reducing the current noise in an external field to equilibrium current fluctuations. We note here that it is actually possible to express $\langle J(t) J(0)\rangle_{E}$ in terms of equilibrium two-point correlation functions. Since

$$
|J(t)|=q a \sum_{i} \delta\left(t-\tau_{i}\right)=\frac{2 q}{a} D(t),
$$

Eq. (8) can be rewritten as

$$
\langle J(t) J(0)\rangle_{E}=\left(\frac{q a E}{2 k T}\right)^{2}\langle|J(t)||J(0)|\rangle \quad(t>0),
$$

where the right-hand side is, as usual, an equilibrium average. This result is simpler than Eq. (25) and, perhaps, more aesthetically pleasing. But it is less general than Eq. (25) since it explicitly involves the lattice constant and thus depends on the existence of a lattice.

For the generation of $1 / f$ noise one needs long-time correlations in the fluctuations of $D(t)$. Actually, noise that varies approximately as $1 / f$ is obtained only if $C_{D}(t)$ is almost constant, typically varying as $\log (t)$ to some negative power. One may imagine two different ways of generating $1 / f$ noise. The one case is that of "genuine" mobility fluctuations, i.e., when the random walk is a doubly stochastic Poisson point process with $1 / f$ noise in the $\widetilde{D}(t)$ fluctuations of Eq. (10). The other case is when long-time correlations in $D(t)$ arise because of occasional long trapping times of the particles. Here one must assume the existence of a broad spectrum of trapping times exceeding the longest experimental times. This may be regarded as the case of number fluctuations since a charge carrier trapped for the whole period of observation for all practical purposes is nonexistent.

We close this section by showing that only $1 / f$ noise with strong fluctuations in the number of jumps is observable. By strong noise we mean noise obeying

$$
\left.\left\langle(\Delta N)^{2}\right\rangle_{t}\right\rangle\langle N\rangle_{t}
$$

on the relevant time scale. This is the criterion for $N$ fluctuations much larger than for an ordinary random walk, where there is equality in Eq. (28) on account of the Poisson statistics. Both the mobility and the trapping mechanism may satisfy Eq. (28) which rules out only the case of very weak mobility fluctuations. To show Eq. (28) we calculate first the white noise in equilibrium. From $J=q a \sum_{i} \pm \delta\left(t-\tau_{i}\right)$ we get immediately

$$
S_{\text {white }, J}(\omega)=2 q^{2} a^{2} \frac{\langle N\rangle_{t}}{t}, \quad t=\omega^{-1}
$$

where the number of jumps per unit time, $\langle N\rangle_{t} / t$, is of course independent of $t$. In a weak external field there is a slight increase in the white noise which, however, is insignificant for the present calculation. ${ }^{22,23}$ Since $C_{D}(t)$ varies only very slowly one has $S_{D}(\omega) \lesssim 4 t C_{D}(t)$ and Eq. (13) implies $C_{D}(t) \simeq\left(a^{4} / 4\right)\left[\left\langle\left(\Delta N^{2}\right)\right\rangle_{t} / t^{2}\right]$, where $t=\omega^{-1}$. Combining these results we get for the excess current noise

$S_{\mathrm{exc}, J}(\omega) \lesssim q^{2} a^{2}\left(\frac{q a E}{k T}\right)^{2} \frac{\left\langle(\Delta N)^{2}\right\rangle_{t}}{t}, t=\omega^{-1}$.

In linear-response theory $q a E / k T<<1$ and the criterion for measurable excess noise reduces to Eq. (28). This condition is independent of the size of the system since both sides of Eq. (28) are additive.

\section{EXAMPLE: EXCESS NOISE IN THE CONTINUOUS-TIME RANDOM-WALK MODEL}

One way or the other $1 / f$ noise arises from long-time correlations in the diffusion constant fluctuations. This may occur, for instance, via occasional very long trapping times for the change carriers. The simplest example of this is the CTRW model of Montroll and Weiss. ${ }^{25}$ Here the jump probability at any time is a function only of the time elapsed since the preceding jump. The CTRW model was first applied to the $1 / f$ noise problem by Tunaley $^{23}$ and later by Nelkin and co-workers. ${ }^{24,26}$ The central quantity in this model is the waiting time distribution function, $\psi(t)$, which is the probability density for jumps the time $t$ after the latest jump. In the language of stochastic point processes, the sequence of waiting times is a so-called renewal process. ${ }^{16,27}$ In this section we calculate the excess noise from $\psi(t)$. This was done by Tunaley ${ }^{23}$ but is repeated here as an illustration of the general theory of Sec. II and also because the below derivation is simpler than that of Tunaley.

If the particle jumps at $t=0$ we let $\psi_{n}(t)$ denote the probability density for the $n$th jump hereafter occurring at time $t$. Obviously one has

$$
\psi_{n+1}(t)=\int_{0}^{t} \psi_{n}(t-\tau) \psi(\tau) d \tau
$$

For the function $f(t)$ defined by

$$
f(t)=\sum_{n=1}^{\infty} \psi_{n}(t)
$$

Eq. (31) implies

$$
f(t)-\psi(t)=\int_{0}^{t} f(t-\tau) \psi(\tau) d \tau .
$$

Taking the Laplace transform of this equation one gets $\widetilde{f}(s)-\widetilde{\psi}(s)=\widetilde{f}(s) \widetilde{\psi}(s)$, or

$$
\widetilde{f}(s)=\frac{\widetilde{\psi}(s)}{1-\widetilde{\psi}(s)}
$$

The quantity $p(t \mid 0)$ occurring in Eq. (9) is just $f(t)$ and thus 


$$
\begin{aligned}
S_{\mathrm{exc}}(\omega) \equiv \frac{S_{\mathrm{exc}, D}(\omega)}{D^{2}} & =\frac{a^{2}}{2 D} 4 \int_{0}^{\infty} f(t) \cos (\omega t) d t \\
& =\frac{2 a^{2}}{D} \operatorname{Re} \widetilde{f}(i \omega) \\
& =\frac{2 a^{2}}{D}\left[\operatorname{Re} \frac{1}{1-\widetilde{\psi}(i \omega)}-1\right] .
\end{aligned}
$$

This is Tunaley's result for the excess noise. ${ }^{23}$ It is convenient to write $\psi(t)$ as a sum of exponential decays, $\psi(t)=\left\langle\gamma e^{-\gamma t}\right\rangle$, where the average is over a distribution of jump rates. ${ }^{24,26}$ From this $\widetilde{\psi}(s)=\langle\gamma /(\gamma+s)\rangle$ and $D=\frac{1}{2} a^{2}\left\langle\gamma^{-1}\right\rangle^{-1}$ which substituted into Eq. (35) yields

$$
\begin{aligned}
S_{\mathrm{exc}}(\omega) & =\frac{2 a^{2}}{D}\left[\operatorname{Re} \frac{1}{i \omega\left\langle\frac{1}{\gamma+i \omega}\right\rangle}-1\right) \\
& =\frac{2 a^{2}}{D \omega}\left(\operatorname{Im}\left\langle\frac{1}{\gamma+i \omega}\right)^{-1}-\omega\right),
\end{aligned}
$$

i.e.,

$$
S_{\text {exc }}(\omega)=\frac{4\left\langle\gamma^{-1}\right\rangle}{\omega}\left(\operatorname{Im}\left\langle\frac{1}{\gamma+i \omega}\right\rangle^{-1}-\omega\right)
$$

A simple example yielding $1 / f$ noise is the case of all $\gamma$ 's equally likely:

$$
p(\gamma)=\frac{1}{\gamma_{0}}, \quad \gamma_{\min }<\gamma<\gamma_{0}
$$

where $\gamma_{\min }<<\gamma_{0}$ is assumed. One may think of $\gamma_{0}$ as a phonon frequency and $\gamma_{\min }$ as corresponding to long waiting times, e.g., one day or one year. For the whole range of intermediate frequencies $\left\langle(\gamma+i \omega)^{-1}\right\rangle$ $\simeq \gamma_{0}^{-1} \ln \left(\gamma_{0} / i \omega\right)$ and

$S_{\mathrm{exc}}(\omega) \simeq \frac{4}{\omega} \ln \lambda \operatorname{Im} \frac{1}{\ln \left(\gamma_{0} / i \omega\right)} \simeq \frac{2 \pi \ln \lambda}{\ln ^{2}\left(\gamma_{0} / \omega\right)} \frac{1}{\omega}$

where $\lambda=\gamma_{0} / \gamma_{\min }$. We thus find $S_{\text {exc }}(\omega) \propto \omega^{-\alpha}$, where

$$
\alpha=1-\frac{2}{\ln \left(\gamma_{0} / \omega\right)}
$$

At ordinary laboratory frequencies one has $\alpha \simeq 0.9$. This model may be termed the "standard model of $1 / f$ noise" since it is probably the simplest exact soluble randomwalk model giving $1 / f$ excess noise. Of course $1 / f$ noise is built into the model via Eq. (38). This is equivalent to a waiting time distribution function $\psi(t) \propto t^{-2}$ (Ref. 28) above $\gamma_{\min }$, which implies a logarithmically divergent average waiting time. The excess noise of the standard model is identical in functional form to the expression derived by Kuzovlev and Bochkov for a scale-invariant random walk. ${ }^{10,20}$ Their derivation is based on Eq. (20) which has been criticized above. The derivation is not quite transparent; also, the factor $\ln \lambda$ in Eq. (39) is absent in their calculation of the excess noise.

\section{DISCUSSION}

Random-walk models provide a simple framework for understanding the fluctuation-dissipation theorem. It is therefore an obvious idea also to use these models for getting a better understanding of low-frequency resistance fluctuations. Various random-walk models have been studied with this purpose. ${ }^{19-24,26,28-30}$ In this paper a general framework for discussing excess noise in random-walk models has been proposed. Following previous work, in Sec. II we focused on the concept of a fluctuating diffusion constant, the dynamical diffusion constant $D(t)$. While the exact definition of $D(t)$ hitherto has not been clear, we here use a definition of $D(t)$ which is simpler than previous implicit definitions. For all practical purposes, however, it is identical to these since the expressions for $\langle D(t) D(0)\rangle$ in Eqs. (19) and (23) are identical to those given by Kuzovlev and Bochkov, ${ }^{19,20}$ and Machta, Nelkin, Nieuwenhuizen, and Ernst. ${ }^{21,22}$ The relevance of $D(t)$ to excess current noise is shown by Eq. (8) according to which the current fluctuations in an external field directly probes the equilibrium $\langle D(t) D(0)\rangle$. An important general property of random-walk models is the fact that the noise is proportional to $\langle J\rangle_{E}^{2}$ and inversely proportional to the volume or any other extensive property like the number of charge carriers. This follows immediately from Eq. (8) since $D(t)$ and autocorrelation functions are additive.

As an application of the general formalism, note that Eq. (6) is valid also in a time-dependent external field. From this it is straightforward to show that in a sinusodial field one finds the so-called $1 / \Delta f$ noise $^{31-33}$ which is directly proportional to the magnitude of the $1 / f$ noise in a constant field. A weakness of the formalism of Sec. II should be mentioned, namely, that it does not easily allow for an exact calculation of the white noise in an external field. Though insignificant in the linear regime, there is an interesting small increase in the white noise when the field is turned on. ${ }^{22,23}$

The dynamical diffusion constant is proportional to the absolute value of the current. This leads to a simple expression for the current fluctuations in an external field in terms of equilibrium fluctuations [Eq. (27)], a result which can be rewritten in terms of autocorrelation functions as

$$
\left.\left\langle\left[J(t)-\langle J\rangle_{E}\right]\left[J(0)-\langle J\rangle_{E}\right]\right\rangle_{E}=\mid \frac{q a E}{2 k T}\right]^{2}\langle[|J(t)|-\langle|J|\rangle][|J(0)|-\langle|J|\rangle]\rangle(t>0)
$$


Analogous to the frequency-dependent conductivity which probes the equilibrium velocity autocorrelation function, the excess-noise spectrum thus probes the equilibrium speed autocorrelation function. But it should be remembered that a frequency-independent conductivity must be assumed to derive Eq. (41), and also that the random walk takes place on a lattice. Equation (41) cannot be expected to apply more generally. For this to be the case, one should be able to define a characteristic length to play the role of the lattice constant $a$ in Eq. (41). The only possibility for this seems to be to let $a$ be the length for which $q a E \simeq k T$ at fields marking the onset of nonlinearities. Since, however, nonlinearities involve new physics which is in general uncorrelated to linearresponse phenomena, there is little hope that this approach can be generally valid. Thus, Eq. (41) must be limited to lattice models.

The unified formalism for excess current noise in random-walk models developed above provides a convenient starting point for a discussion of general properties of $1 / f$ noise. In particular, the point process approach makes it possible to throw some light on the old controversy as to whether $1 / f$ noise is due to mobility or to number fluctuations. ${ }^{6,9}$ We end this paper by giving a general argument to the effect that Fermi statistics rules out the number-fluctuation mechanism. Mobility fluctuations correspond to noise in the effective charge-carrier Hamiltonian, whereas number fluctuations due to occasional deep trapping is noise generated by the Hamiltonian itself. In the language of point processes, the standard example of mobility fluctuations is the case when the jump times $\tau_{i}$ constitute a doubly stochastic Poisson process, while the standard example of trapping noise is the case when $\tau_{i}$ is a renewal process, i.e., when we have a continuous-time random-walk. This case is nonMarkovian and therefore in a sense unphysical, admittedly, but the CTRW model is equivalent to a Markovian multistate trapping model where the noise then is indeed generated by the Hamiltonian. ${ }^{26}$

In Sec. III the excess noise in the CTRW model was calculated. A simple example termed the standard model was worked out in detail. This model is one out of the class of CTRW models discussed by Nelkin and Harrison. ${ }^{26}$ In the multistate trapping realization of the CTRW, the standard model corresponds to a density of energies, $\varepsilon$, given by

$$
p(\varepsilon) \propto \exp (\varepsilon / k T) .
$$

Though this implies only very few deep trapping states, in the course of time all energies are equally likely to be occupied because of the Boltzmann factor to be multiplied with Eq. (42) to get the probability. Thus, the standard model is just another example of the old idea of $1 / f$ noise arising when all activation energies are equally likely. At the same time the standard model satisfies the requirement for $1 / f$ noise given by Nieuwenhuizen and Ernst, namely, an exponential density of states. ${ }^{22,28}$

A temperature-dependent density of states as in Eq. (42) arises in a system of fermions. Here a single particle senses, in a mean-field approximation, a density of avail- able states below the Fermi energy, $p_{\text {eff }}(\varepsilon)$, which is given by

$$
p_{\text {eff }}(\varepsilon) \simeq n(\varepsilon) e^{\left(\varepsilon-\varepsilon_{F}\right) / k T} \quad\left(\varepsilon<\varepsilon_{F}\right),
$$

where $n(\varepsilon)$ is the ordinary density of states. For a constant $n(\varepsilon)$ Eq. (42) is obtained. Equation (43) implies $1 / f$ noise in the motion of the single particles, a point we will return to below. While interesting on its own, this has nothing to do with the observed $1 / f$ resistance fluctuations, however. This is because the mean-field approximation does not apply due to strong interparticle correlations: Below the Fermi level the particle number fluctuations (in one energy level) are exponentially small, $\left\langle(\Delta n)^{2}\right\rangle=\langle n\rangle\langle 1-n\rangle \ll\langle n\rangle$ (Ref. 34), while for independent particles one would have $\left\langle(\Delta n)^{2}\right\rangle=\langle n\rangle$ because of the Poisson statistics, i.e., much larger fluctuations.

We now extend the above and argue that Fermi statistics actually rules out any $1 / f$ noise model based on the trapping mechanism. Basically, one may imagine two different ways of producing long trapping times. The first case involves hopping between localized states of similar energy separated by various long tunneling distances or large energy barriers. In this case, inevitably, there is a strong frequency dependence of the conductivity and models of this kind cannot explain the usual case of $1 / f$ fluctuations of a frequency-independent conductivity. (Note that McWhorter's model based on tunneling to surface states ${ }^{9}$ is not ruled out by this argument which is only concerned with bulk and isotropic $1 / f$ noise.) The second way of having long trapping times is that of trapping into deep energies. This only works for independent particles with the peculiar density-of-states of Eq. (42). For fermions, the density-of-states above the Fermi energy is not temperature dependent, and below the Fermi level, where Eq. (43) does apply in a mean-field sense, the particles are not independent as discussed already.

To summarize the effect of Fermi statistics, it has been argued that for Fermi systems $1 / f$ resistance fluctuations cannot be due to occasional deep trapping of the charge carriers. The number-fluctuation mechanism thus can be ruled out on general grounds, and, e.g., the CTRW model in the multiple trapping realization is not realistic. Thus, the noise must be caused by mobility fluctuations. The central problem, which until today remains largely unsolved, is to identify the origin of the mobility fluctuations.

While Fermi statistics rules out number fluctuations, it implies on the other hand a peculiar kind of "singleparticle" $1 / f$ noise: The motion of a single localized fermion exhibits $1 / f$ noise as a consequence of Eq. (43). In principle this is observable, since, for a system of fermions described by a master equation (an implicit assumption in the argument), there are no quantum coherence effects and, in effect, the particles are classical and distinguishable. Similarly, atoms or ions diffusing in a disordered medium with a distribution of available potential minima also exhibit single-particle $1 / f$ noise, since they behave as fermions because of their strong repulsion. This should be observable by monitoring the motion of tracer atoms or ions in time. 
Except for the above arguments ruling out number fluctuations, the cause of $1 / f$ noise has not been discussed in the present paper. We feel there is no simple and generally valid mechanism explaining $1 / f$ noise. Rather, $1 / f$ noise may arise from a number of different mechanisms which probably, one way or the other, involve lattice defects overcoming or tunneling through barriers. Following this line of thought, $1 / f$ noise and its dependence on temperature, etc., provide important information about the solid under study. Note that while $S_{\text {exc }}(\omega)$ probes only $\langle D(t) D(0)\rangle$ there is an enormous amount of information hidden in the higher-order correlations of $D(t)$. This is illustrated by the fact that the total amount of information in the noise is contained in a function of two variables

$$
P(u, t)=\left\langle e^{u N}\right\rangle_{t},
$$

while $S_{\text {exc }}(\omega)$ is only a function of one variable. The fact that there is more to the noise than just the spectrum was emphasized already ten years ago by Voss in a discussion of linearity of the $1 / f$ noise mechanism. ${ }^{35}$ The higherorder correlations of $J(t)$ and thereby $D(t)$ are accessible today by digital techniques and their determination should provide a means of distinguishing between various models for $1 / f$ noise. Other promising lines of research is the measurement of $1 / f$ noise anisotropy, ${ }^{11}$ and the problem of stationarity of the noise and the dependence of the noise on the annealing state (in any system with long relaxation times the possibility of "glass transitions" should not be forgotten); $1 / f$ noise is still mainly an experimental field and it seems that a lot of work remains to be done here before reliable theories can be proposed.

\section{ACKNOWLEDGMENT}

This work was supported by the Danish Natural Science Research Council.
${ }^{1}$ H. Bittel, Ergeb. Exakten Naturwiss. 31, 84 (1959).

${ }^{2}$ F. N. Hooge, Physica B + C 83B, 14 (1976).

${ }^{3}$ D. Wolf, in Noise in Physical Systems, edited by D. Wolf (Springer-Verlag, Berlin, 1978), p. 122.

${ }^{4}$ W. H. Press, Comments Astrophys. Space Phys. 7, 103 (1978).

${ }^{5}$ A. van der Ziel, Adv. Electron. Electron Phys. 49, 225 (1979).

${ }^{6}$ D. A. Bell, J. Phys. C 13, 4425 (1980).

${ }^{7}$ P. Dutta and P. M. Horn, Rev. Mod. Phys. 53, 497 (1981).

${ }^{8}$ K. M. van Vliet and H. Menta, Phys. Status Solidi B 106, 11 (1981).

${ }^{9}$ F. N. Hooge, T. G. M. Kleinpenning, and L. K. J. Vandamme, Rep. Prog. Phys. 44, 479 (1981).

${ }^{10}$ G. N. Bochkov and Y. E. Kuzovlev, Usp. Fiz. Nauk 141, 151 (1983) [Sov. Phys. - Usp. 26, 829 (1983)].

${ }^{11}$ S. M. Kogan Usp. Fiz. Nauk. 145, 285 (1985) [Sov. Phys. Usp. 28, 170 (1985)].

${ }^{12}$ R. F. Voss and J. Clarke, Phys. Rev. B 13, 556 (1976).

${ }^{13}$ M. A. Mikulinsky, Phys. Lett. 66A, 440 (1978).

${ }^{14}$ A.-M.S. Tremblay and M. Nelkin, Phys. Rev. B 24, 2551 (1981).

15J. K. E. Tunaley, J. Appl. Phys. 43, 3851 (1972).

${ }^{16}$ S. K. Srinivasan, Stochastic Point Processes (Griffin, London, 1974).

${ }^{17}$ D. L. Snyder, Random Point Processes (Wiley, New York, 1975).

${ }^{18}$ B. Saleh, Photoelectron Statistics (Springer-Verlag, Berlin,
1978)

${ }^{19}$ Y. E. Kuzovlev and G. N. Bochkov, Pis'ma Zh. Tekh. Fiz. 8, 1260 (1982) [Sov. Tech. Phys. Lett. 8, 542 (1982)].

${ }^{20}$ Y. E. Kuzovlev and G. N. Bochkov, Radiophys. Quantum Electron. 26, 228 (1983).

${ }^{21}$ J. Machta, M. Nelkin, T. M. Nieuwenhuizen, and M. H. Ernst, Phys. Rev. B 31, 7636 (1985).

${ }^{22}$ T. M. Nieuwenhuizen and M. H. Ernst, J. Stat. Phys. 41, 773 (1985).

${ }^{23}$ J. K. E. Tunaley, J. Stat. Phys. 15, 149 (1976).

${ }^{24}$ C. J. Stanton and M. Nelkin, J. Stat. Phys. 37, 1 (1984).

${ }^{25}$ E. W. Montroll and G. H. Weiss, J. Math. Phys. 6, 167 (1965).

${ }^{26}$ M. Nelkin and A. K. Harrison, Phys. Rev. B 26, 6696 (1982).

${ }^{27}$ D. R. Cox, Renewal Theory (Methuen, London, 1962).

${ }^{28}$ T. M. Nieuwenhuizen and M. H. Ernst, Phys. Rev. B 33, 2824 (1986).

${ }^{29}$ H. Spohn, Z. Phys. B 57, 255 (1984).

${ }^{30}$ W. Lehr, J. Machta, and M. Nelkin, J. Stat. Phys. 36, 15 (1984).

${ }^{31}$ B. K. Jones and J. D. Francis, J. Phys. D 8, 1172 (1975).

${ }^{32}$ R. F. Voss and J. Clarke, Phys. Rev. Lett. 36, 42 (1976).

${ }^{33}$ M. A. Mikulinsky, Phys. Lett. 71A, 473 (1979).

${ }^{34}$ L. D. Landau and E. M. Lifshitz, Statistical Physics (Pergamon, Oxford, 1969).

${ }^{35}$ R. F. Voss, Phys. Rev. Lett. 40, 903 (1978). 